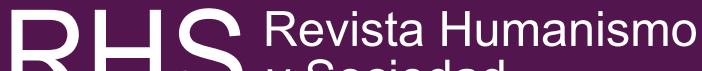 $R H S_{\text {rsocectac }}$
}

RHS. Revista. Humanismo. Soc. 8(2): 132 - 145, diciembre 2020 / ISSNe 2339-4196

\section{Reto de las mipymes para incursionar en el marketing digital en tiempos de COVID-19}

Challenges faced by MSMEs to get into digital marketing in times of COVID-19
Sandra P. Ochoa Guevara'

sandra.ochoa@sena.edu.co

sandrapatricia8a@gmail.com

https://orcid.org/0000-0001-6089-1229

César Medina Acero ${ }^{1}$

cmacero@sena.edu.co

https://orcid.org/0000-0002-2434-6669

Lina Y. Santamaría Burgos ${ }^{1}$

santameria@sena.edu.co

linayibi@yahoo.com

https://orcid-org/0000-0001-8013-9982

Deily I. Ramírez Campos ${ }^{1}$

diramirez@sena.edu.co

rcislen@gmail.com

https://orcid.org/0000-0002-3121-8358

Edward A. Paramo Renza'

eparamo@sena.edu.co

Edward.paramo@hotmail.com

https://orcid.org/0000-0003-2243-7281

Nancy E. Ochoa Guevara ${ }^{2}$

nancyochoa@unitec.edu.co

ochoaguevara@gmail.com

https://0000-0002-4533-4990

https://doi.org/10.22209/rhs.v8n2a09

1 Servicio Nacional de Aprendizaje SENA Centro de Virtualización Gestión de Mercados, Logística y Tecnologías de la Información. Bogotá, Colombia.

2 Corporación Universitaria UNITEC. Bogotá, Colombia y Corporación Universitaria Remington, Sede Bogotá, Colombia.
Recibido: julio 6 de 2020.

Aceptado: noviembre 30 de 2020

\section{R e s u m e n}

Este artículo describe la crisis que las micro, pequeñas y medianas empresas han experimentado en todo el país durante la emergencia sanitaria (COVID-19), a raíz de la baja producción y la reducción en las ventas. Se busca observar los efectos de la incorporación del marketing digital empresarial que se convierte en un mecanismo para solucionar la problemática y para romper la brecha digital en estas empresas, entendida desde la diferencia socioeconómica entre aquellas comunidades que tienen accesibilidad a las tecnologías de la información y la comunicación y aquellas que no. Para la compilación de información se emplea un análisis exploratorio, comparativo y descriptivo de las principales investigaciones sobre la relación entre marketing digital empresarial y el rendimiento obtenido en el desarrollo empresarial, compromiso de los trabajadores, posicionamiento, satisfacción del consumidor y rendimiento financiero; además los autores de este estudio plasman sus criterios para contribuir a eliminar esta brecha digital.

Los resultados demuestran que, a pesar de la existencia de grandes divergencias, las empresas que hoy debido a la crisis están tratando de mostrar su capacidad tecnológica digital y de conectividad van a ser beneficiadas con un aumento en sus 
ventas y su sostenibilidad en el mercado con una prospectiva internacional.

Palabras clave: tecnología digital; conectividad; reconocimiento; trabajadores; satisfacción del consumidor; reconocimiento empresarial; rendimiento financiero.

\section{Abstrac}

This article describes the crisis that micro, small and medium enterprises have experienced throughout the country during the health emergency (COVID-19), due to low production and reduced sales. It aims to examine the effects of incorporating digital business marketing that becomes a mechanism to deal with this problem and to fill the digital gap of these companies. This issue is addressed considering the socioeconomic difference between those communities that have access to information and communication technologies and those that do not. In order to collect information, an exploratory comparative descriptive analysis was carried out of the main research studies on the relationship between business digital marketing and the outcomes in business development, employee commitment, positioning, consumer satisfaction and financial performance. Additionally, the authors propose criteria to help bridge this digital gap.

The findings show that, despite the existence of great divergences, the companies that today, in face of the current crisis, attempt to leverage their digital technology and connectivity capacity are going to be benefited by an increase in sales and market sustainability with international exposure.

Keywords: digital technology, connectivity, recognition, workers, consumer satisfaction, business recognition, financial performance.

\section{Introducción}

Desde los contextos sociales se determina el comportamiento de los agentes económicos de cualquier economía del mundo, sin importar las condiciones socioeconómicas, culturales, religiosas o de etnias, entre otras, a las que pertenece. La crisis sanitaria y preventiva que enfrenta el mundo por medio de la pandemia ocasionada por la COVID-19 que, según la Organización Mundial de la Salud (OSM) (2020) presentó su primera aparición en la ciudad de Wuhan en China el 31 de diciembre de 2019 (párr. 1) y en Colombia el 11 de marzo de 2020, ha ocasionado, como mencionan Restrepo y Gómez (2020), el aumento de la brecha digital, entendida desde la diferencia socioeconómica entre aquellas comunidades que tienen accesibilidad a las tecnologías de la información y la comunicación (TIC) y aquellas que no; al igual que las diferencias que hay entre grupos según su capacidad para utilizar las TIC de forma eficaz, debido a los distintos niveles de alfabetización y capacidad.

Esta situación no solo ha logrado afectar la salud de las personas, sino también el funcionamiento de las empresas colombianas (Bermeo-Giraldo et al., 2020), en especial las conformadas con la Ley 905 de 2004 -por medio de la cual se modifica la Ley 590 de 2000-, sobre la promoción del desarrollo de la micro, pequeña y mediana empresa llamadas mipymes, dedicadas a la oferta, bienes y servicios en el país. De acuerdo con la Cámara de Comercio de Bogotá (CCB) (2020), estas empresas se caracterizan por el número de empleados (NE) y el valor de sus activos en salarios mínimos mensuales legales vigentes (SMMLV) como la micro: con menos de $10 \mathrm{NE}$ y un activo menor a 500 SMMLV; las pequeñas: entre 11-50 NE y un activos entre 501-5000 
SMMLV; y medianas entre 51-200 NE y un activos entre 5001-3000 SMMLV.

De acuerdo con el Ministerio de Trabajo (2019, párr. 1), estas mipymes representan más del $90 \%$ del sector productivo nacional, generan el $35 \%$ del Producto Interno Bruto (PIB) y el $80 \%$ del empleo en Colombia, datos arrojados por el Departamento Nacional de Estadísticas (DANE) (2017) en la Encuesta de Desarrollo e Innovación Tecnológica (EDIT); sustentada por el Consejo Nacional de Política Económica y Social (CONPES) (2019) como política colombiana Nro. 3956 / 2019.

Según la encuesta de desempeño empresarial realizada por la Asociación Colombiana de las Micro, Pequeñas y Medianas Empresas [ACOPI] (2019) el 14\% (15.274) de un total de 109.104 estimado de las mipymes ha invertido en tecnología digital y de telecomunicaciones. Sin embargo, el Ministerio de Tecnologías de la Información y las Comunicaciones (MINTIC) (2019) en su informe conectividad y transferencia digital resalta que el $74 \%$ de estas mipymes colombianas están conectadas por lo menos a Internet, pero solo el $6 \%$ lo han aprovechado. Ante esta situación las mipymes requieren adaptar el marketing digital empresarial (en adelante MDE), no sin antes medir las consecuencias que esta acción tendrá en su desarrollo empresarial, compromiso de los trabajadores, posicionamiento, satisfacción del consumidor y rendimiento financiero.

De ahí que resulte importante indagar sobre cuáles son las estrategias para llevar a cabo de manera exitosa la incorporación de este MDE en las mipymes colombianas en tiempos de pandemia; con el propósito es observar los efectos de la incorporación del MDE desde el rendimiento empresarial, compromiso de los trabajadores, satisfacción del consumidor y rendimiento financiero. Con el apoyo de una metodología de revisión de fuentes bibliográficas con referentes nacionales e internacionales; por medio de un modelo de revisión documental con un tipo de estudio exploratorio y comparativo bajo cinco componentes como desarrollo empresarial, trabajadores, posicionamiento, satisfacción del consumidor y rendimiento financiero que permiten relacionar e identificar aspectos relevantes en el MDE (Hernández et al., 2014). Estos componentes están centrados desde el mapa de procesos empresariales con el nivel estratégico, misional y de apoyo, es decir, desde la dirección y gerencia, los operativos con los trabajadores o colaboradores, y los agentes funcionales respectivamente, que contribuyen a que estos procesos empresariales se cumplan satisfactoriamente.

\section{Metodología}

Esta investigación se dirige hacia las fuentes bibliográficas con referentes nacionales e internacionales, por medio de un modelo de revisión documental; donde posteriormente se hace un análisis comprensivo e interpretativo, se logra cumplir el objetivo general de la misma y dar respuesta a la pregunta de investigación planteada: ¿cuáles son las estrategias para llevar a cabo de manera exitosa la incorporación de este MDE en las mipymes colombianas en tiempos de pandemia?: 


\section{MDE y desarrollo empresarial}

El MDE se destaca, a mediados de los años 90, como estrategia de innovación también llamado marketing online (en línea) como un «marketing directo que se alimenta de los rápidos adelantos en las tecnologías de bases de datos y los nuevos medios de marketing -especialmente internet-» (Kotler, 2011, p. 599), como Foncillas-García (2017) ratifica que son tendencia al comportamiento del consumidor, al uso y accesibilidad de los contenidos; por tanto en esta época existe infinitos conceptos sobre el MDE, todos se encaminan en el uso de la tecnología digital y de las telecomunicaciones, por medio de la Internet e Intranet, buscando cumplir con la usabilidad y accesibilidad, como lo expresa Marín (2017) y confirmado por los autores de este estudio, resaltando que son muchos los investigadores que se han centrado en investigar realmente el compromiso del MDE en el interior de las mipymes.

Así, por ejemplo, Sosa y Useche (2017) presentan factores para adaptar herramientas de software y hardware libre (free) necesarios para que estas microempresas aprovechen este MDE en este tiempo de COVID-19; aunque el uso de este tipo de herramientas va en aumento por el manejo de computadores, aplicaciones y teléfonos móviles entre otros, ven en la Internet una gran ayuda para dar a conocer sus productos y relacionarse con el cliente y el rendimiento empresarial (Quiroga-Juárez et al., 2015). Pero aun así la incorporación de esta tecnología digital requiere de inversión y capacitación inmediata a sus trabajadores para contrarrestar esta crisis de pandemia (Cano-Pita, 2018) que exige, como lo ratifican los autores del estudio, un sustento económico apropiado para tal fin.
Aunque el Informe Mundial de Tecnologías de la Información refleja que aún existen limitaciones en las mipymes para la adopción de las TIC; situando a Colombia en el lugar número 57 de 141 países del ranking global en el Foro Económico Mundial (FEM) (2019).

También, como lo menciona Ibarra et al. (2017) en su estudio con 3000 mipymes, concluyen que el MDE tiene un fuerte impacto para que estas microempresas puedan avanzar en el entorno digital y mantenerse en el sector empresarial, logrando sostener su mapa de procesos en los niveles estratégicos, misional y de apoyo (Almonacid et al., 2015).

En un estudio realizado por Serna-Gomez et al., (2020) resaltan que en las mipymes de Colombia, el $26,3 \%$ tiene acceso a internet, el 5,7\% tiene redes sociales, el 7,4\% realiza comercio electrónico y el 10,9\% compra de proveeduría; de ahí que se evidencie una cifra alarmante para que estos comercios den el salto hacia la digitalización de sus negocios. Por tanto, se espera que el Gobierno brinde incentivos para que los empresarios logren el sustento de sus negocios con el manejo y uso de los medios y mediaciones digitales como páginas web, redes sociales, mensajerías de texto o aplicaciones, entre otros, indispensables en la estructura organizativa de la empresa para su respectivo posicionamiento de su marca.

Como se ha podido apreciar no existe un consenso sobre si el llevar a cabo una estrategia de MDE conduce a las mipymes a una valiosa salida para mantenerse en el mercado. Nuestro resultado coincide con el afirmado por otros investigadores -Moreno et al. (2017)-, en un estudio realizado en el Ecuador tras analizar la influencia del MDE sobre el resultado de una encuesta a 1800 microempresas. En este 
se deduce que la relación entre ambas magnitudes va a depender mucho de las distintas dimensiones en las que se lleve a cabo el desarrollo de este MDE por tanto Navarre-Torres y Muñoz-Aparicio (2017) resaltan que parte de esta relación y adaptación está en la cultura del empresario que aún no asimila el potencial del uso de la tecnología digital como redes sociales, Youtube, plataformas libres, herramientas de programación libres, BigData, IoT entre otros. Sohangir et al. (2018) mencionan que todavía hay mucho miedo en el uso y aplicación de estos medios digitales. Esto explicaría el porqué de las grandes diferencias mostradas por los diversos investigadores analizados en este estudio.

De igual manera, en la mipymes se reconocen factores claves que viabilizan la capacidad de innovación a la que puede llegar una empresa. Por ejemplo, para Ramos et al. (2018) en su estudio dedicado a 96 pymes de la cadena de valor agroindustrial del departamento del Atlántico en Colombia, determina que los factores de la capacidad de adquisición, barreras externas, ubicación geográfica con proveedores y clientes posibilitan el nivel de innovación de las mismas; muy similar al Ministerio de Comercio, Industria y Turismo (2018) en el informe de dinámicas de producción y el comercio exterior basado en la economía del conocimiento y la gran capacidad de relacionamiento que poseen las pequeñas empresas. Lo cual permite sustentar que una plataforma de MDE le ofrece a las mipymes la posibilidad de un desarrollo empresarial reconocido en el mercado.

De la misma manera, Pérez y Maldonado (2019) en su evaluación, realizada a 321 pymes de las ciudades de Armenia, Barranquilla, Bogotá, Bucaramanga, Cali, Manizales, Medellín y Pereira en Colombia, sobre el análisis organizacional, los procesos y las herramientas de tecnología de información y las comunicaciones, para gestionar conocimiento, identificaron que 2,2/5,0 de las pymes no usan TIC para la generación de conocimiento y para enfrentar escenarios de competitividad. Los autores recomiendan la incorporación de herramientas tecnológicas y digitales para lograr identificar, compartir, generar, retener y aplicar un nuevo conocimiento en el desarrollo e innovación de productos y, así, poder reducir la brecha digital en las mipymes, motivándolas a estar a la vanguardia de las tecnologías y sus propietarios en constante capacitación, esto les permitirá estar inmersos en procesos de mejora continua.

Por tanto, en la mayoría de los estudios del MDE para la transformación de las mipymes en Colombia, se resalta la incorporación de estas herramientas tecnológicas y digitales definido por Martínez-López et al. (2020) como el conjunto de páginas web desarrolladas en código HTML en un dominio de Internet World Wide Web (www). De ahí la importancia de referenciar algunas de estas herramientas digitales como el diseño de Blog, conceptualizado por Ferrer y Martínez (2019) visto desde una página en un sitio web en la que se puede publicar información personal y comentar sobre la misma; de igual forma, las Redes Sociales (Facebook, Twitter, YouTube, Linked In, Google Plus) definidas, según los investigadores de este estudio, como un espacio creado virtualmente para facilitar la interacción entre personas con fines parecidos, donde se encuentran herramientas para ayudar a las mipymes a evolucionar e incorporar sus actividades económicas hacia la digitalización e internacionalización.

Esta transformación, a partir de las herramientas digitales y las diversas estrategias 
para ofrecer más servicios y productos digitales y físicos, se considera desde hace algunos años sumamente importante para poder incursionar y sobrevivir en mercados internacionales, dado que las compañías en el área digital son las más dinámicas y las que más rápidamente crecen (Panandiker et al. 2018, p. 10; MGI 2017, p 11).

\section{MDE y compromiso}

\section{de los trabajadores}

No cabe duda, como lo concluye Aguilera et al. (2017), que el mayor valor que tienen las mipymes son sus trabajadores desde la prospectiva de la funcionalidad en la producción local e internacional, de ahí se espera que mediante este MDE se establezcan cursos de capacitación y actualización en herramientas digitales y de telecomunicación de última generación, como lo expresan Bischhoffshausen et al. (2015), para lograr la motivación y compromiso Institucional. Acorde con esto, algunos investigadores han trato de analizar la relación entre el MDE y el compromiso de los trabajadores con sus microempresas.

La mayoría de estos investigadores ha hecho énfasis en la percepción que los trabajadores de las mipymes, que son el factor fundamental de ventas y accionar empresarial, tienen sobre el uso y aplicación de las tecnologías digitales y de telecomunicación que se viene desarrollando en el interior de la micro empresa (González, 2020). Así, Weller (2020) resalta que debido a la crisis actual que están sufriendo las empresas por la COVID-19 en especial las mipymes; han tomado algunas represiones laborales hacia sus trabajadores realizando cambios día a día y bajando sus ingresos, al igual que crece la desigualdad de oportunidades laborales. También se resalta que esta crisis puede llevar a altos niveles de desconfianza e incertidumbre en el puesto de trabajo, por parte del personal y sobre todo desajustes salariales como lo evidencia la Oficina Internacional del Trabajo (OIT) (2020) en su informe mundial de salarios y desigualdad de ingresos; donde más de 25 millones buscan empleo y no lo consiguen; al igual que otros elementos encontrados similar a los hallados por Tundidor, Nogueira, Medina y Serrte (2018), como la desinformación de los colaboradores, la resistencia al cambio de los propietarios y la falta de profesionalización en MDE construido a la medida para las mipymes de este estudio; esto hace complejo avanzar en la gestión organizacional y sobre todo en la incorporación de tecnologías digitales para fortalecer su gestión y productividad.

Por otro lado, Lovelock y Wirtz (2015) analizan los efectos del MDE en el comportamiento de sus colaboradores o trabajadores en países occidentales. Los autores encuentran una relación significativa de los trabajadores con una actitud positiva en el manejo y adaptación de tecnologías digitales y de telecomunicación para el desarrollo y ejecución de las tareas asignadas en sus puestos de trabajo. Similar al estudio de prácticas o ejercicios en MDE Tundidor et al. (2018) donde demuestran que el uso y manejo de plataformas en el MDE responsables y competitivas conlleva implicaciones positivas en el compromiso de los trabajadores de estas mipymes. Estos resultados están en consonancia con los divulgados por Navarrete-Torres, Muñoz-Aparicio (2017), quien tras una exhaustiva revisión teórica y de investigaciones previas sobre la relación entre la MDE y el capital intelectual, concluye la existencia de una fuerte relación entre ambas 
variables teniendo por tanto el MDE, como principal objetivo para mejorar la actividad empresarial de las mipymes, que operan en todos los sectores económicos del país.

\section{MDE y posicionamiento} empresarial

Gómez y Borda (2018) resaltan que el comercio cada vez está sufriendo cambios abruptos sobre sus actividades económicas e incluso el funcionamiento de las diferentes empresas, independiente de su tamaño, antigüedad o solvencia está siendo sometida a cambios significativos; Esto se evidencia en la encuesta de la Red de Cámaras de Comercio en Colombia (CONFECAMARAS) (2020) aplicada a 20.035 empresarios de mipymes de 691 municipios de Colombia, muy similar a los resultados presentes de la Asociación Colombiana de las Micro, Pequeñas y Medianas Empresas (ACOPI) (2020) en la «Encuesta de desempeño empresarial» aplicada a 272 mipymes del sector de la manufactura, quienes manifestaron la necesidad de implementar tecnologías digitales de telecomunicación como respuesta a la crisis y de paso incursionar en la revolución industrial mediante los servicios virtuales.

De igual forma, el autor Horna-Ysla (2017) estudia de qué manera el MDE se correlaciona con el posicionamiento de las microempresas; resalta que el consumidor de hoy se informa, decide y adquiere los productos a la medida, a través de diversos canales o medios; así, logra esta incidencia correlacional con la aplicación de herramientas tecnológicas especializadas como Marketing Viral, SEM, Podcasting, Publicidad contextual, SEO, e-Auctions, e-Games,
VRM, e. Commerce y Avatar Marketing entre otros, que contribuyen a fortalecer la calidad de los productos en un mercado cambiante.

Esto ha dado lugar a que muchos estudios se centren en la correlación de estas dos variables MED y su influencia en el posicionamiento empresarial, como sucedió en la investigación realizada en Cuba por Pérez, Ramírez y Topete (2017) con una muestra de 1034 mipymes, donde las prácticas de MDE aumentaron el reconocimiento y posicionamiento en estas microempresas; al igual que otro estudio comparativo de Torres et al. (2017), en el que se logra medir la efectividad en el posicionamiento que las mipymes han logrado con el aumento de sus ventas y el reconocimiento de sus clientes mediante publicidad virtual y la comunicación digital. Sin embargo, los autores de esta investigación resaltan que aun estas variables están afectadas por la brecha digital que sigue existiendo en el uso de las herramientas digitales por parte de estas empresas (Restrepo y Gómez, 2020).

Santillán y Rojas (2017) elaboraron un plan de negocio utilizando el marketing digital como elemento esencial en el posicionamiento de la empresa con base a las opiniones de los consumidores potenciales y posteriormente realizaron una entrevista a 35 expertos, en la que analizaron la relación entre las variables MDE y el posicionamiento empresarial. Santillán y Rojas, al igual que Marín-López y Robledo (2017), concluyen que existe una fuerte relación entre estas variables y que, además, este posicionamiento ejerce un efecto de mediación total en la relación entre la MED y los resultados económicos, lo que tiene importantes implicaciones directivas sobre la gestión del MED desde una perspectiva estratégica. 


\section{MDE y satisfacción del consumidor}

Muchas investigaciones se han centrado en analizar la relación entre el MDE y la evaluación que los consumidores hacen de las mipymes y las marcas de productos. Olmedo (2015), en su investigación «Marketing 4.0», identifica las herramientas digitales relevantes para que el consumidor logre hacer negocio, en este caso el cliente es quien tiene el control y no la empresa; muy similar a la posición de Rojas-Reyes et al. (2018), quienes concluye que la influencia del MDE en las mipymes ha encontrado un apoyo positivo en la satisfacción de los consumidores, por medio de las redes sociales donde se resalta el beneficio e impacto en las ventas y compras de sus productos.

Por su parte, Contreras et al. (2018) corroboran que el MDE tiene una proporción directa con el consumidor, y llegan a la conclusión que estas herramientas digitales como las redes, e-comercio, email Marketing, herramienta para creación de Landing Pages, Plataforma de Marketing de Contenido, herramienta de SEO (Search Engine Optimization / Optimización en Buscadores), herramienta de Analytics y CMS (Sistema de Gestión de Contenidos) entre otros, tiene un proceso automático identificando alertas tempranas en el estado continuo del negocio empresarial; como Peralta et al. (2020) que determinaron el tiempo de valoración que tienen para los consumidores el requisito y envío de la información por estos medio digitales a satisfacción personal, lo cual contribuye a mejorar la imagen de marca, con los resultados positivos que esto produce en el medio y largo plazo. Estas apreciaciones coinciden con los aportados por Mañez (2017), los cuales confirman como las acciones de negocio por el MDE posicionan y satisfacen a los usuarios en las empresas. Este es el propósito con el que Sohangir et al. (2018) buscan diferencias en el punto de vista de los consumidores, encontrando una fuerte correlación entre el ejercicio de las prácticas en el MDE y la orientación del mercado hacia estas mipymes.

Estas herramientas digitales se consideran una variable relevante en relación con el MDE, pues despiertan en el consumidor un nivel de lealtad y motivación hacia la empresa. Así, por ejemplo, Espinosa y Maldonado (2018) analizan los efectos que las prácticas del MDE generan en la lealtad de los consumidores en el sector de la líneas de ropa deportiva de marca, concluyen que, aunque existe un importante espacio para la mejora, los consumidores son conscientes y valoran muy positivamente el marketing digital al hacer uso de redes sociales, el e-comercio, email-marketing y otros; como lo ratifican Stocker et al. (2019) para la oferta y conocimiento de sus productos, donde se busca estudiar el perfil de los consumidores.

\section{MDE y rendimiento financiero}

Otros investigadores, como Correa et al. (2018) relacionan los efectos del manejo y uso de estas herramientas digitales como una práctica de apoyo al MDE, lo que permite una línea ascendente en el rendimiento financiero de las mipymes, en un momento en que la revolución digital está cambiando la forma acelerada en que se vive, trabaja y consume, al ofrecer la oportunidad de la aplicación de estas herramientas digitales como valor agregado a las empresas, aun más en esta crisis causada 
por la pandemia. Por tanto, ante la realidad es cada vez más creciente la presión sobre los directivos de marketing digital para medir el verdadero valor añadido de las partidas asignadas a sus presupuestos.

Pero Gálvez-Albarracín et al. (2018) resaltan que las mipymes colombianas se financian con recursos propios más que con los externos, como se logra observar en la «Encuesta de desarrollo e innovación tecnológica» (EDIT) del DANE para los años 2015-2016, realizada con 8610 empresas industriales de todos los tamaños. Esta señala que en Colombia $74,7 \%$ de ellas son no innovadoras, $3,6 \%$ potencialmente innovadoras, $21,5 \%$ innovadoras en sentido amplio, y apenas $0,2 \%$ innovadoras en sentido estricto. Asimismo, que $56,7 \%$ de las innovaciones que realizaron corresponden a métodos de producción y logística, $24,7 \%$ a nuevos métodos organizativos y $18,6 \%$ a nuevas técnicas de comercialización; por su parte, las mayores limitaciones que encontraron las empresas que quisieron innovar, pero no pudieron hacerlo fueron la escasez de recursos propios (42,1\%), la dificultad para acceder al financiamiento externo $(29,6 \%)$ y la facilidad de imitación por terceros $(26,7 \%)$. Por último, indica que las principales fuentes de financiación usadas fueron en su orden: recursos propios, banca privada, otras empresas del grupo, recursos públicos, otras empresas, fondos de capital privado y cooperación o donaciones (DANE, 2017), y como expresan Prandhan et al. (2018), puede ser por las dificultades para acceder a créditos bancarios, obstáculos para comprender las prácticas de financiamiento o simplemente por el desconocimiento.

Otras entidades que apoyan a las mipymes no solo en el aspecto financiero, sino también técnico, es el caso de Bancolombia (2020) con el programa "Manufactura avanzada e industrias 4.0" facilitando la adaptación de las tecnologías para el sector industrial de estas mipymes; similar a De Miquel (2020) cuando resalta la creación del Centro de Transformación Digital Empresarial (CTDE) por parte de MinTIC, como asistencia técnica para el proceso de apropiación estratégica de las tecnologías para aumentar su competitividad e ingreso a la internacionalización.

Motivado por encontrar una relación entre MDE y la financiación de las mipymes, González (2019) analiza la configuración de un modelo de previsión, para refinar inversiones y focalizarlas en generar un tráfico de mayor calidad que fortalezca el desempeño financiero de las empresas colombianas y encuentran una relación positiva y significativa. Siguiendo un camino muy similar, en un estudio sobre las microempresas de un país en desarrollo Marín y López (2020), con base en los resultados obtenidos, recomiendan a las mipymes realizar un esfuerzo en materia de implantación del MDE, ya que esto reportará mayores ventajas a la hora de acceder a una financiación nacional e internacional.

Por tanto, ACOPI (2019) sustenta que el $72 \%$ de las mipymes no poseen estrategias para iniciar procesos de exportación en razón a que los aspectos financieros y de logística los limita, aún más frente a la emergencia sanitaria. Caso similar al que comentan López et al. (2018), donde manifiestan que Colombia creó el programa «Fabricas de Productividad» por medio del cual las mipymes pueden recibir asistencia técnica para mejorar su productividad y competitividad. Por tanto el programa de la Organización para la Cooperación y el Desarrollo Económicos (OCDE), resalta que las mipymes deben ser la prioridad para la política pública en toda América Latina y el Caribe. 
De igual forma Peralta et al. (2020) y Perdigón (2018) concluyen que el MDE de genera elementos de valor agregado competitivos en diversos sectores como la publicidad digital en un estudio realizado en Barranquilla, Colombia, visto desde una perspectiva transaccional en los ingresos derivados del consumidor; coinciden, a la vez, con Limas Suárez et al. (2019) en los resultados de productos de la línea cosmética en Boyacá, Colombia. La relación de estas dos variables MDE y rendimiento financiero son, por lo tanto, rentables para invertir en negocios que se puedan proporcionar en el futuro en el tiempo de pandemia.

\section{Conclusiones}

A partir de este estudio exploratorio realizado, se resalta la necesidad de efectuar un análisis para resolver muchas dudas y romper los mitos que se tiene sobre la incorporación del MDE en un gran número de mipymes en Colombia, mostrando los resultados positivos y significativos que arrojó este estudio con las variables de desarrollo empresarial, compromiso de los trabajadores, posicionamiento empresarial, satisfacción del consumidor y rendimiento financiero. Finalmente, se identifica el MDE como una de las mejores opciones en tiempos de COVID-19, fortaleciendo el éxito de su implementación en las mipymes.

En esta dinámica, los resultados sugieren que las prácticas o ejercicios del MED deben estar soportados, desde las mipymes, para lograr un aumento significantico en su posicionamiento, desde la inversión, motivación y capacitación a sus trabajadores. Además de la incorporación de tecnologías digitales de última generación como las redes sociales, Youtube, plataformas libres, herramientas de programación libres, BigData, loT, entre otras, según la recomendación de los autores Sohangir, Pomeranets y Khoshgoftaar (2018). De esta manera se logra romper los mitos de «miedo» y se asegura la incorporación de herramientas digitales seguras con un alto nivel de rendimiento y tiempos de respuestas inmediatos, lo que le permite a las empresas -sin importar su tamaño, sector, ciudad o país centrar sus esfuerzos en contribuir con la sociedad a superar las crisis en el aspecto social, económico y sanitario, entre otros.

Asimismo, es una estructura de estudio que permite ser base para otros tipos de investigaciones explicativas de la realidad encontrada, tomando elementos, indicadores o componentes para medir o comparar diversos métodos o procedimientos que estén afectando los resultados de dicha realidad. De igual manera, se fortalece la posibilidad para que Representantes Legales o Gerentes de las empresas que estén presentando resultados bajos o negativos en su accionar, puedan superar la crisis en su entorno económico sanitario.

Por último, esta investigación invita a las mipymes a que revisen los bajos niveles que tienen en cuanto al uso y aplicación de tecnologías digitales para el ofrecimiento de un marketing digital a la medida, con el fin de establecer nuevas estrategias en estos tiempos de pandemia; de igual forma, a las universidades para que aumenten sus esfuerzos en la formación de capital intelectual de alto nivel en el manejo y aplicación del MDE para todo tipo de negocio. 


\section{Referencias}

- Aguilera-Castro, A., Ávila-Fajardo, G., \& SolanoRodríguez, O. (2017). Las TIC en la formulación estratégica de las pymes de Santiago de Cali Colombia. Entramado, 13(1), 102-111. https://doi. org/10.18041/entramado.2017v13n1.25106

- Almonacid-Paredes, L., \& Herrera-Gallardo A. (2015). Estrategias de Marketing digital y su influencia en el posicionamiento de la empresa MCH GRUPO INMOBILIARIO S.A.C. en el distrito de Trujillo 2015 [Tesis de pregrado, Universidad Privada Antenor Orrego]. https://bit.ly/31cvZmU

- Asociación Colombiana de las Micro Pequeñas y Medianas Empresas [ACOPI]. (2019). Encuesta de desempeño empresarial. 2do trimestre de 2019. Encuesta de Desempeño Empresarial, (10) https://bit.ly/3i8wRi1

- Asociación Colombiana de las Micro Pequeñas y Medianas Empresas [ACOPI]. (2020). Encuesta de desempeño empresarial. 1er trimestre 2020. Encuesta de Desempeño Empresarial,(13). https://bit.ly/3m0vj0x

- Bancolombia, (2020). Programas de apoyo del Gobierno a pymes e incentivos no monetarios. https://bit.ly/3hYlvNG

- Bermeo-Giraldo, M.C., Montoya-Restrepo, L. A., Valencia-Arias, A., y Mejía Cardona, M.A. (2020). Incursión de las TIC en la gestión de la información financiera en las empresas pyme comerciales: estudio de caso. NOVUM, 1(10), 2541. https://bit.ly/306jQ20

- Cámara de Comercio de Bogotá. (2020). Acompañamiento en Convivencia Empresarial. https://bit.ly/357SWsG

- Cano-Pita, G. (2018). Las TICs en las empresas: evolución de la tecnología y cambio estructural en las organizaciones. Dominio de las Ciencias, 4(1), 499-510. https://doi.org/10.23857/ dc.v4i1.762
- Consejo Nacional de Política Económica y Social. CONPES (2019). Como política de formalización empresarial Número 3956/2019 desde el Departamento Nacional de Planeación en Colombia. https://bit.ly/305AxLh

- Contreras Monroy, S. R., \& Peñaloza Suarez, L. (2018). El Talento Humano y el cuidado ambiental en la industria hotelera. Turismo y Sociedad, 23, 85-100. https://doi.org/10.18601/01207555. n23.05

- Correa, F., Dini, M., \& Stumpo, G. (2018). El rol de las MiPymes y las políticas de fomento: algunas conclusiones. En: M. Dini y G. Stumpo. (Coords.), MIPYMES en América Latina Un frágil desempeño y nuevos desafíos para las políticas de fomento (pp. 547-557). Comisión Económica para América Latina y el Caribe (CEPAL).

- De Miquel, E. F. (2020). La transformación digital en el centro veterinario, Anestesia y analgesia Argos, (218), 14-15.

- Departamento Nacional de Estadísticas (DANE). (2017). Encuesta de Desarrollo e Innovación Tecnológica [EDIT]. https://bit.ly/2IA6CoJ

- Espinosa, T., Maldonado, G., \& Uc Heredia, L. (2018). Los ingresos familiares como una fuente de financiamiento de los micro negocios de mujeres de la península de Yucatán, México. TEC Empresarial, 12(1), 31-38. https://doi. org/10.18845/te.v12i1.3569

- Fernández, N. G., Moreno, M. L. R., \& Guerra, J. R. (2020). Brecha digital en tiempo del COVID-19. Revista Educativa HEKADEMOS, (28), 76-85.

- Ferrer Fernández, A., \& Rosabal Martínez, A. (2019). Blogs interactivos como herramientas en las escuelas deportivas (Original). Revista Científica Olimpia, 16(54). https://bit.ly/334Hu12

- Foncillas-Garcia B. (2017). El sector financiero ante el reto digital. Revista de Economía ICE, (898), 23-34. https://bit.ly/2HeSXTf

- Foro Económico Mundial (FEM). (2019). WEF -Informe Global de tecnología de la información. https://bit.ly/3kGZlf8 
- Gálvez-Albarracín, E., Mayorga Sánchez, J., \& Chávez Hernández, A. (2019). Impacto del autofinanciamiento sobre la innovación de las micro, pequeñas y medianas empresas colombianas. Criterio Libre, 16(29), 121-139. https://doi.org/fdpz

- Gómez, H., \& Borda, S. (2018). Diagnóstico del micro, pequeñas y medianas empresas: políticas e instituciones de fomento en Colombia. En M. Dini y G. Stumpo. (Coords.), MIPYMES en América Latina: Un frágil desempeño y nuevos desafíos para las políticas de fomento (pp. 227288). Comisión Económica para América Latina y el Caribe (CEPAL).

- González Ordóñez, A. I. (2019). Gestión ambiental y competitividad de las PYMES del sector comercio en el cantón Machala, Provincia El Oro, Ecuador. Revista Espacios, 40(27). https://bit. ly/3kSz2YP

- González Ordóñez, A. I. (2020). La formación ambiental del talento humano y la competitividad en las mipymes de los sectores agropecuario, servicios y comercio, provincia El Oro, Ecuador. Revista Científica Agroecosistemas, 8(1), 15-22. https://bit.ly/362qzyi

- (2014). Metodología de la Investigación (6. a ed.). Mc Graw Hill Education.

- Horna-Ysla, J. (2017). Marketing digital y su relación con el posicionamiento en los clientes de las pastelerías Mypes del distrito de Trujillo, en el año 2017 [Tesis de Maestría en Administración de Negocios - MBA, Universidad César Vallejo, Trujillo]. https://bit.ly/3nX0Y08

- Ibarra Cisneros, M. A., González Torres, L. A., \& Demuner Flores, M. del R. (2017). Competitividad empresarial de las pequeñas y medianas empresas manufactureras de Baja California. Estudios fronterizos, 18(35), 107-130. https://doi.org/10.21670/ref.2017.35.a06

- Kotler, P. \& Armstrong, G. (2011). Principios de Marketing (12. ${ }^{\text {a ed.). }}$ Pearson Education.
- Limas Suárez, S. J., Vargas Soracá, G., \& Salazar Araujo, E. J. (2019). La comunicación corporativa y su influencia a través de la publicidad digital. Un estudio en productos de la línea cosmética y cuidado corporal en el departamento de Boyacá (Colombia). RISTI, (E20), 126-138. https://bit. Iy/2G02kGm

- López, J. M., Somohano, F. M., \& Martínez, F. J. (2018). Efecto de la innovación en la rentabilidad de las MIPYMES en contextos económicos de recesión y expansión. TEC Empresarial, 12 (1), 7-18. https://doi.org/10.18845/te.v12i1.3567

- Lovelock, C., \& Wirtz, J. (2015). Marketing de Servicios. Personal, tecnología y estrategia (7. a ed.). Pearson. Educación.

- Marín-López, J. C., Robledo, J. S., \& DuqueMéndez, N. (2017). Marketing Emprendedor: Una Perspectiva Cronológica Utilizando Tree of Science. Revista Civilizar de Empresa y Economía, 7(13), 113-123. https://bit.ly/3i9YRSM

- Marín López, J. C., \& López Trujillo, M. (2020). Análisis de datos para el marketing digital emprendedor. Caso de estudio del Parque de Innovación Empresarial de Manizales. Universidad \& Empresa, 22(38), 65-78. https:// doi.org/d98p

- Mañez, R. (12 marzo, 2017). La captación de clientes por medio de las redes sociales. Nuestras mejores consultas del Marketing. https://bit.ly/2TOWAiv

- Maldonado Córdova, A. V., \& Pérez Espinoza, M. J. (2019). El Talento Humano: la clave del éxito para el mejoramiento de la competitividad de las empresas. Revista Científica Agroecosistemas, 7(1), 68-74. https://bit.ly/3kRSYLp

- Martínez-López, F. J., Anaya, R., Aguilar, R., \& Molinillo, S. (2015). Online Brand Communities: Using the Social Web for Branding and Marketing. Springer.

- Mejía, J. (2018). Definición del marketing digital, su importancia y principales estrategias. https:// www.juancmejia.com/ 
- Ministerio de trabajo. (2020). Mipymes representan más de $90 \%$ del sector productivo nacional y generan el $80 \%$ del empleo en Colombia. https://bit.ly/2Ew7Lw1

- Ministerio de Tecnologías de la Información y las Comunicaciones (MINTIC). (2015). Informe conectividad y transformación digital. El 74\% de las MiPymes colombianas están conectadas a Internet. https://bit.ly/37dlysX

- Ministerio de Comercio, Industria y Turismo (2018). Informe dinámicas de producción y comercio exterior de pymes manufactureras. Programa de Transformación Productiva. Recuperado el 1 de noviembre de 2018 de: https://bit.ly/342bGZY.

- Moreno Sotomayor G.R, Ruiz Dávila, D. C., \& Peña herrera Pereira L. V. (2017). Influencia del marketing digital en el uso de las redes sociales para la gestión empresarial de las pymes. Revista ARJÉ, 11(21), 271-279. https://bit.ly/2RY2Kzh

- Navarrete-Torres M., Muñoz-Aparicio C. (2017). Marketing digital para los consumidores del siglo XXI. https://bit.ly/3nXxyPx

- Organización Mundial de la Salud (2020) Neumonía de causa desconocida - China. https://bit.ly/3/14BA7.

- Oficina Internacional del Trabajo (OIT). (2020). Informe Mundial sobre Salarios: Salarios y desigualdad de ingresos. OIT. https://bit. Iy/3nXY9fo

- Olmedo-Altamirano A. (2015). El Marketing Digital y su influencia en la Captación de clientes para la empresa GOLOSITORTIZ de la ciudad de Ambato. [Tesis de pregrado en Marketing y Gestión de Negocios, Universidad Técnica de Ambato en Ecuador]. https://bit.ly/3IXSggv

- Peralta Miranda, P., Salazar-Araujo, E., Álvarez Mendoza, Z. V., \& Ortiz Cardona, J. (2020). Marketing digital, elementos de la cadena de valor generadores de competitividad del sector publicitario en Barranquilla (Colombia). RISTI, (E27), 201-212. https://bit.ly/3mRxHDE
- Pérez Rendón, L., Ramírez Segovia, N., Topete Gómez, E. (2017). Estudio comparativo del uso de tecnologías de información y comunicación en pymes y grandes empresas. Revista RAITES, 3(7), 49-74. https://bit.ly/3cy0R68

- Panandiker R., Waltermann, B., Maggard, K., Aureliano, C., Azevedo, D., Chan, T., \& Kuipers, H. (2018). The 2018 BCG Local Dynamos Emerging-Market Companies Up Their Game. The Boston Consulting Group.

- Perdigón Llanes, R., Viltres Sala, H., \& Madrigal Leiva, I. R. (2018). Estrategias de comercio electrónico y marketing digital para pequeñas y medianas empresas. Revista Cubana de Ciencias Informáticas, 12(3), 192-208.

- Prandhan, P., Nigam, D., \& Tiwari, C. (2018). Digital marketing and SMES: An identification of research gap via archives of past research. Journal of Internet Banking and Commerce, 1(23), 1-14. https://bit.ly/2yzQNqr

- Quiroga-Juárez, C. A., \& Villalobos-Escobedo, A. (2015). Análisis del comportamiento bursátil de las principales bolsas financieras en el mundo usando el análisis multivariado (análisis de componentes principales PCA) para el periodo de 2011 a 2014. Revista CEA, 1(2), 25-36. https:// doi.org/10.22430/24223182.122

- Ramos Ruiz, J. L., Polo Otero, J. L., Arrieta Barcasnegras, A. A., \& Vélez Durán, L. F. (2018). Determinantes del grado de apertura de las pymes agroindustriales: una aplicación para el departamento del Atlántico. Revista Desarrollo y Sociedad, (80), 189-229.

- Red de Cámaras de Comercio (CONFECAMARAS). (2019). DINÁMICA DE CREACIÓN DE EMPRESAS EN COLOMBIA Enerodiciembre de 2019. https://bit.ly/2G7JoVN

- Restrepo Tamayo, J. F., \& Gómez Agudelo, D. S. (2020). La conectividad digital como derecho fundamental en Colombia. Revista de Direito, Estado e Telecomunicações, 12(1), 113-136. https://doi.org/10.26512/Istr.v12i1.31161 
- Rojas-Reyes, R., Vilchez-Paz, R., \& Bernita, S. (2018). Gestión del talento humano y su relación con el desempeño laboral del personal del puesto de salud Sagrado Corazón de Jesús [Tesis de maestría en Gestión de la Salud, Universidad Norbert Wiener]. https://bit. ly/358S9b5

- Ruiz-Marquina, J. F. (2018). Decisiones de Financiamiento y el Desarrollo Empresarial de las Empresas Panetoneras. [Tesis de pregrado en Contaduría Pública, Universidad César Vallejo, Perú]. https://hdl.handle. net/20.500.12692/23572

- Santillán Garay, J., Rojas Herrera, S. (2017). El marketing digital y la gestión de relaciones con los clientes (CRM) de la Empresa Manufacturas Kukuli S.A.C., 2017 [Título de pregrado, Universidad Nacional José Faustino Sánchez Carrión]. https://bit.ly/2GXTvNW

- Santes Sosa, R. M., Navarrete Torres, M. del C., y Garcia Muñoz A. C. (2017). Marketing digital para los consumidores del siglo XXI. Hitos De Ciencias Económico Administrativas, 23(65), 24-37.

- Serna-Gómez, H. M., Barrera-Escobar, A., \& Castro-Escobar, E. S. (2020). Efectos en el empleo en los micros y pequeñas empresas generados por la emergencia del COVID-19: Caso Colombia. ORMET, Universidad de Manizales. https://doi.org/10.2139/ssrn.3609574

- Sohangir, S., Wang, D., Pomeranets, A., \& Khoshgoftaar, T. M. (2018). Big Data: Deep Learning for Financial Sentiment Analysis. Journal of Big Data, 5(1). https://doi.org/10.1186/ s40537-017-0111-6
- Sosa Tota, A. S., \& Useche Aguirre, M. C. (2017). Marketing digital en universidades privadas en el estado Zulia. Poliantea, 13(24), 6-26. http://doi. org/10.15765/plnt.v13i24.1001

- Torres, R., Rivera, J., Cabarcas, R., \& castro, T. Y. (2017). The effectiveness of the use of digital marketing as a strategy for the positioning of PyMes for the trade sector in the city of Barranquilla. Investigación y desarrollo en TIC, 8(2), 5-9. https://bit.ly/2MfFill

- Tundidor-montes de Oca, L., Nogueira-Rivera, D., Medina-León, A., \& Serrate-Alfonso, A. (2018). Requerimientos de los sistemas informativos para potenciar el control de gestión empresarial. Ciencias Holguín, 24(1), 43-56.

- Tundidor Montes de Oca, L., Nogueira Rivera, D., \& Medina León, A. (2018). Organización de los sistemas informativos para potenciar el control de gestión empresarial. Cofín Habana, 12(1), 88110. https://bit.ly/3400JZ1

- Von Bischhoffshausen, J. K., Paatsch, M., Reuter, M., Satzger, G., \& Fromm, H. (2015). An Information System for Sales Team Assignments Utilizing Predictive and Prescriptive Analytics.

2015 IEEE 17th Conference on Business Informatics, 1, 68-76. https://doi.org/10.1109/ CBI.2015.38

- Stocker, F., Mascena, K., Cunha, M., Azevedo, A. C., Boaventura, J., \& Gama, M. (2019). Teoría de redes de influencia de «stakeholders»: un enfoque revisado. Cad. EBAPE.BR, 17, 673-688. http://doi.org/10.1590/1679-395176683

- Weller, J. (2020). La pandemia del COVID-19 y su efecto en las tendencias de los mercados laborales. CEPAL. 